\title{
Method of Moments Enhancement Technique for the Analysis of Sierpinski Pre-Fractal Antennas
}

\author{
Josep Parrón, Jordi Romeu, Member, IEEE, Juan M. Rius, Member, IEEE, and Juan R. Mosig, Fellow, IEEE
}

\begin{abstract}
The numerical analysis of highly iterated Sierpinski microstrip patch antennas by method of moments (MoM) involves many tiny subdomain basis functions, resulting in a very large number of unknowns. The Sierpinski pre-fractal can be defined by an iterated function system (IFS). As a consequence, the geometry has a multilevel structure with many equal subdomains. This property, together with a multilevel matrix decomposition algorithm (MLMDA) implementation in which the MLMDA blocks are equal to the IFS generating shape, is used to reduce the computational cost of the frequency analysis of a Sierpinski based structure.
\end{abstract}

Index Terms-Antennas, fractals, integral equation, method of moments (MoM).

\section{INTRODUCTION}

$\mathrm{N}^{\mathrm{T}} \mathrm{u}$ TRUCTURES based on the Sierpinski fractal are particularly interesting due to their multiband behavior [1], [2]. The radiation parameters of the antenna can be numerically computed using integral equation methods discretized by method of moments (MoM) [3].

In the case of highly iterated pre-fractal structures, there are many small geometry details that require tiny MoM subdomain basis functions for an accurate discretization of the induced current. This, together with the fact that the multiband antenna is electrically large at the highest operating bands, leads to a very large number of unknows $(N)$ in the MoM formulation. The computational requirements to solve the full linear system using conventional methods (memory increases as $N^{2}$ and CPU time as $N^{3}$ ) can easily overcome the capabilities of desktop computer systems.

This paper will tackle the optimization of the MoM solution taking advantage of the geometrical properties of the iterated function system (IFS) [10] that generates the antenna geometry. Since the IFS is inherently multilevel, the most suitable MoM acceleration algorithms here are multilevel domain subdivision methods that can use Rao, Wilton, and Glisson linear triangle basis functions (RWG) [9], namely the multilevel fast

\footnotetext{
Manuscript received January 10, 2002; revised July 26, 2002. This work was supported by the "Departament d'Universitats Recerca i Societat de la Informació (DURSI)" of the "Generalitat de Catalunya" under "Distinció de la Generalitat de Catalunya per a la Promoció de la Recerca Universitaria," the Spanish "Comisión Interministerial de Ciencia y Tecnología (CICYT)" through grant TIC 2001-2364-C01-01 and the European Commission through FET project IST-2001-33055.

J. Parrón, J. Romeu, and J. M. Rius are with the Signal Theory and Communications Department, Universitat Politècnica de Catalunya Jordi Girona 1-3, Mòdul D4, 08034 Barcelona, Spain (e-mail: parron@tsc.upc.es).

J. Mosig is with the Laboratory of Electromagnetics and Acoustics, École Polytechnique Fédérale de Lausanne LEMA, EL-B, EPFL, Ecublens, CH1015 Lausanne, Switzerland (e-mail: Juan.Mosig@epfl.ch).

Digital Object Identifier 10.1109/TSP.2003.815428
}

multipole algorithm (MLFMA) [6] and the multilevel matrix decomposition algorithm (MLMDA) [7], [8]. This paper will present results only for the MLMDA, but the optimization strategies introduced here can be easily implemented also in the MLFMA.

\section{IFS}

Like most pre-fractal structures, the Sierpinski antenna can be built by using the concept of IFS [10]. Every IFS iteration is defined by a set of $Q$ affine transformations in the plane $\left\{\omega_{q}\right\}_{q=1}^{Q}: R^{2} \rightarrow R^{2}$ which can be written as

$$
\begin{aligned}
\omega_{q}(x)= & A_{q} x+t_{q} \\
= & \left(\begin{array}{cc}
r_{q 1} \cos \theta_{q 1} & -r_{q 2} \sin \theta_{q 2} \\
r_{q 1} \sin \theta_{q 1} & r_{q 2} \cos \theta_{q 2}
\end{array}\right)\left(\begin{array}{l}
x_{1} \\
x_{2}
\end{array}\right) \\
& +\left(\begin{array}{c}
t_{q 1} \\
t_{q 2}
\end{array}\right)
\end{aligned}
$$

where $x_{1}$ and $x_{2}$ are the coordinates of point $x$. If $x_{1}$ and $x_{2}$ are the coordinates of point $x$. If $r_{q 1}=r_{q 2}=r_{q}$, with $0<r_{q}<1$, and $\theta_{q 1}=\theta_{q 2}=\theta_{q}$, the IFS transformation is a contractive similarity (angles are preserved) where $r_{q}$ is the scale factor and $\theta_{q}$ is the rotation angle. The column matrix $t_{q}$ is just a translation on the plane. Fig. 1 shows the Sierpinski fractal obtained from a single triangle after applying a set of transformations recursively.

Structures generated by an IFS necessarily have many equal subdomains at different levels. The impedance matrix resulting from MoM discretization has therefore plenty of redundant information, since the interaction between equal pairs of subdomains produces equal submatrices (1A, 2A, 3A in Fig. 2) if the Green's function has translation symmetry, as is the case here. There are many sets of equal submatrices in $[Z]$ at different levels. The MoM implementation presented here uses the IFS definition in order to avoid recomputation and storage of redundant matrix elements.

\section{Multilevel Algorithms}

The electric field integral equation (EFIE) in the frequency domain discretized by MoM may be expressed in matrix form as [3], [9]

$$
-\left[E_{i}\right]=[Z][J]
$$

where $[J]$ are the coefficients of the induced current discretized in RWG basis functions (unknowns), $\left[E_{i}\right]$ is the discretization of the incident field and $[Z]$ is the impedance matrix. This matrix includes the Green's function with all the information about the multilayer media. 


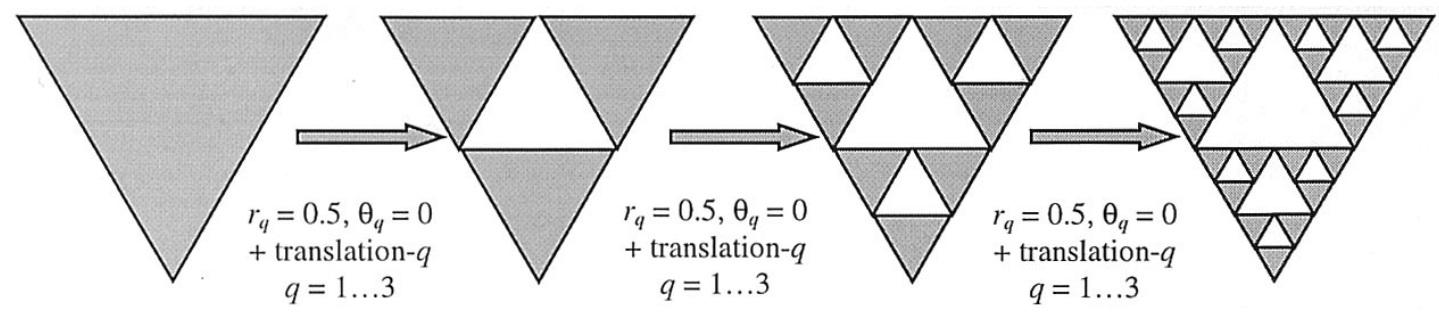

Fig. 1. Four iteration Sierpinski fractal obtained after a set of affine transformations.

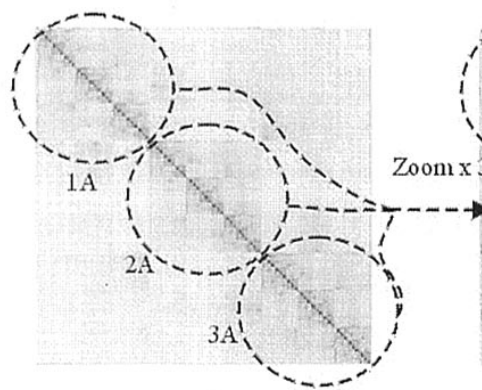

A)

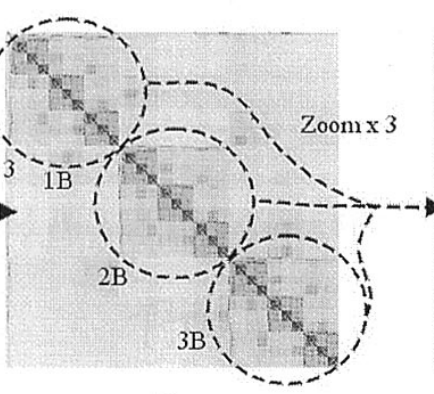

B)

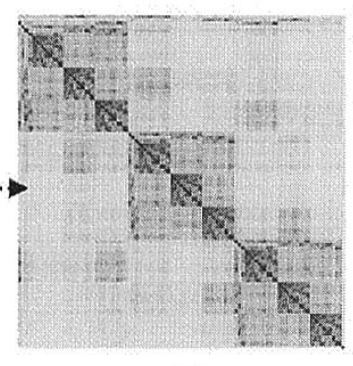

C)

Fig. 2. Method of moments impedance matrix for a five iteration Sierpinski patch antenna. Grey scale shows the magnitude of the matrix elements. If the structure is generated by an IFS, the impedance matrix has plenty of redundant information: submatrices $1 \mathrm{~A}, 2 \mathrm{~A}$ and $3 \mathrm{~A}$ are identical. Besides, due to the recursive application of the IFS, each one of these submatrices has also redundant information, as shown in B and C.

The induced current coefficients $[J]$ are found using the Generalized Minimum Residual (GMRES) [4] iterative method. In each iteration, the main computational effort to obtain the $k$ th estimation of the induced current $\left[J^{(k)}\right]$ are the matrix-vector products $[Z]\left[J^{k-1)}\right]$. Using direct matrix-vector multiplication, the operation count and the memory requirements for each iteration are proportional to $N^{2}$.

\section{A. Multilevel Subdivision of the Object}

In order to reduce the operation count in the direct matrixvector multiplication from $N^{2}$ to $N \log N$, the MLMDA and the MLFMA divide the object into an octal tree in three dimensions or a quad tree in two dimensions containing many nonoverlaping subdomains or boxes. The quad tree domain subdivision that is generally used for arbitrary structures is applied here to a Sierpkinski fractal in Fig. 3.

The interaction between a pair of subdomains can be computed as

$$
\left[E_{m}\right]=\left[Z_{m n}\right]\left[J_{n}\right]
$$

where $n$ and $m$, respectively, are the indexes of the RWG basis functions in the source and observation subdomains and $\left[Z_{m n}\right]$ is a submatrix of the impedance matrix. If all possible pairs of subdomains are considered, the matrix vector multiplication $[Z]\left[J^{(k-1)}\right]$ may be obtained as the addition of submatrix operations of the form (3).

\section{B. $M L M D A$}

The MLMDA will be only outlined here, more details can be found in [8]. It consists of a recursive procedure that begins at level 2 and stops at the finest level $L$. For each nonempty source and observation boxes which belong to the same subdivision level $l(2 \leq l \leq L)$ there are two possible cases:

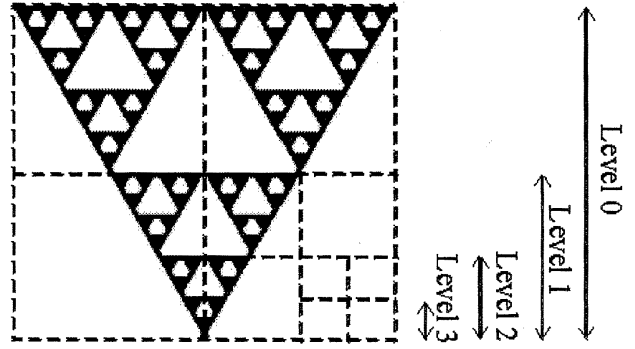

Fig. 3. Multilevel decomposition of the box enclosing a Sierpinski fractal in square subdomains.

1) Boxes are touching one another or are the same: then they are subdivided into level $l+1$ boxes, except if we have already reached the finest level, $l=L$. If this is the case, direct submatrix-vector multiplication (3) is performed, requiring the previous computation of the corresponding matrix terms of $\left[Z_{m n}\right]$. Time and memory requirements are proportional to $M_{b} N_{b}$ where $M_{b}$ and $N_{b}$ are the number of original RWG functions in the source and observation box, respectively.

2) Boxes are not touching each other: In this case, the number of degrees of freedom (DoF) of the electric field $\left[E_{m}\right]$ at the observation box in (3) is smaller than $N_{b}$, and decreases for larger box-to-box distance and for smaller box size [7], [8]. Therefore, $\left[E_{m}\right]$ in (3) can be computed very efficiently in much less than $M_{b} N_{b}$ operations by replacing the original currents by a very small set of equivalent ones that radiate the same field.

The computational cost of this recursive algorithm increases with the number of nonempty boxes in the multilevel subdivision of a given object. If we realize that boxes do not need to be square, as in Fig. 3, while they do not overlap and cover the whole geometry of the antenna, we can use boxes of the same 
TABLE I

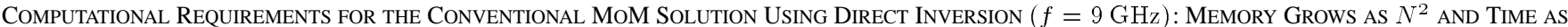

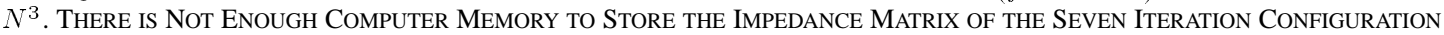

\begin{tabular}{cccccc}
\hline & \multicolumn{2}{c}{ Building $[Z]$} & Direct inversion & Total & Total \\
\cline { 2 - 4 } & Mem. $(\mathrm{MB})$ & $\mathrm{t}(\mathrm{s})$ & $\mathrm{t}(\mathrm{s})$ & Mem. $(\mathrm{MB})$ & Time $(\mathrm{s})$ \\
\hline 4 it., $\mathrm{N}=568$ & 4.9 & 3 & 1 & 4.9 & 4 \\
5 it., $\mathrm{N}=1702$ & 44.2 & 21 & 14 & 44.2 & 35 \\
6 it., $\mathrm{N}=5104$ & 397.5 & 176 & 383 & 397.5 & 559 \\
7 it., $\mathrm{N}=15310$ & 3577.5 & --- & -- & 3577.5 & -- \\
\hline
\end{tabular}

TABLE II

Computational Requirements For the MoM Solution Using the GMRES Iterative Method $(f=9 \mathrm{GHz})$. Time Performance Has IMPRoved, HOWEVER the SEVEn ItERATION CONFIGURATION CANNOT Be ANALYZED Yet

\begin{tabular}{|c|c|c|c|c|c|c|c|c|c|}
\hline & \multicolumn{2}{|c|}{ Building $[Z]$} & \multicolumn{2}{|c|}{ Preconditioning } & \multicolumn{3}{|c|}{ Iteration } & \multirow{2}{*}{$\begin{array}{c}\text { Total } \\
\text { Mem.(MB) }\end{array}$} & \multirow{2}{*}{$\begin{array}{c}\text { Total } \\
\text { Time (s) }\end{array}$} \\
\hline & $\operatorname{Mem}(\mathrm{MB})$ & $t(s)$ & Mem(MB) & $t(s)$ & n. it & $\mathrm{t}$ per it(s) & It.time (s) & & \\
\hline 4 it., $N=568$ & 4.9 & 3 & 0.4 & 0.2 & 5 & 0.3 & 1.5 & 5.3 & 5 \\
\hline 5 it., $N=1702$ & 44.2 & 21 & 3.8 & 2 & 4 & 0.6 & 2.4 & 48.0 & 25 \\
\hline 6 it., $N=5104$ & 397.5 & 176 & 27.3 & 25 & 3 & 6.0 & 18 & 424.8 & 219 \\
\hline 7 it, $\mathrm{N}=15310$ & 3577.5 & -- & -.. & $-\cdots$ & --- & -- & --- & --- & -- \\
\hline
\end{tabular}

TABLE III

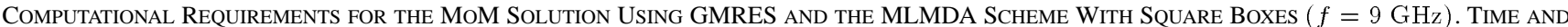
Memory Requirements are Smaller Than the Two Previous Strategies, SPecially When $N$ Grows

\begin{tabular}{cccccccccc}
\hline & \multicolumn{2}{c}{ Building [Z] } & \multicolumn{2}{c}{ Preconditioning } & \multicolumn{2}{c}{ Iteration } & Total & Total \\
& Mem(MB) & $\mathrm{t}(\mathrm{s})$ & $\mathrm{Mem}(\mathrm{MB})$ & $\mathrm{t}(\mathrm{s})$ & $\mathrm{n}$. it & $\mathrm{t}$ per it(s) & It.time (s) & Mem.(MB) & Time (s) \\
\hline 4 it., $\mathrm{N}=568$ & 3.7 & 3 & 0.4 & 0.2 & 5 & 0.1 & 0.5 & 4.1 & 3.7 \\
5 it., $\mathrm{N}=1702$ & 25.3 & 12 & 3.8 & 1.5 & 4 & 0.3 & 1.2 & 29.1 & 15 \\
6 it., $\mathrm{N}=5104$ & 102.1 & 50 & 27.3 & 17 & 3 & 1.5 & 4.5 & 129.4 & 71 \\
7 it, $\mathrm{N}=15310$ & 375.1 & 184 & 128.3 & 136 & 9 & 8 & 72 & 503.4 & 392 \\
\hline
\end{tabular}

shape and size as the IFS building blocks. For the case of the Sierpinski antenna the optimum choice is triangular boxes. This results in a multilevel subdivision with: a) many empty boxes and b) many pairs of source and field boxes having the same interaction matrices either in step 1 or in the equivalent source formulation of step 2. These matrices will be computed once, stored in memory and reused whenever required, leading to a dramatic reduction in computation time and memory storage.

\section{RESULTS}

Configurations of a microstrip Sierpinski patch antenna with four, five, six, and seven iterations have been used as a benchmark in order to test different approaches to enhance the MoM analysis. In all the cases the scale factor is 2 and the height of the equilateral triangle defining the Sierpinski patch (level 0 in Fig. 3) is $8.89 \mathrm{~cm}$. The dielectric substrate is $1.57 \mathrm{~mm}$ thick with a relative permitivity of 2.33 . The patch was excited with a standard coaxial probe located in the lower corner of the Sierpinski fractal.

The computer used in the simulation is a desktop PC with an AMD Athlon CPU at $1.33 \mathrm{GHz}$ and $1.5 \mathrm{~GB}$ of RAM. The programming language is MATLAB 6 with time-critical routines coded in $\mathrm{C}$ language.

Table I shows the computational requirements for the solution of the MoM linear system (2) using direct matrix inversion. Memory requirements grow as $N^{2}$ and time as $N^{3}$, as expected. For the seven iteration configuration, the storage requirements overcome the available memory.
In Table II the same test is repeated using GMRES instead of the direct inversion. Preconditioning is used to reduce the number of iterations [4]. The preconditioner is an sparse matrix that includes all the impedance matrix elements corresponding to basis and testing functions that are close to each other, and zeros elsewhere. The incomplete LU decomposition [4] allows fast multiplication by the inverse of the preconditioner at each itaration. The extra time used in the preconditioning and the small extra increase in memory are more than compensated by the very small number of iterations. The time for building the matrix remains the same as before, while frequently the GMRES iterative solution is one order of magnitude faster. It is therefore the matrix filling time which now becomes the bottleneck.

The MLMDA scheme can be used to compute the matrix vector multiplication $[Z]\left[J^{(k-1)}\right]$ in GMRES, instead of building the whole $[Z]$ matrix. Table III shows the computational requirements of MLMDA with the commonly used square box subdivision (Fig. 3). The matrix filling time and the iteration time are greatly reduced, since MLMDA does not need to compute explicitily all the elements of $[Z]$. This reduction is particularly important for the large problem of 15310 unknowns, in which the $\operatorname{MoM}[Z]$ matrix does not fit in computer memory.

The last level of optimization is reached when triangular boxes are used in the MLMDA approach and IFS symmetries are exploited in order to avoid recomputation of redundant MLMDA matrices (Table IV). Memory requirements increase only linearly with the number of unknowns, while the CPU time per iteration increases roughly as $N \log N$. Matrix filling is no more the slowest part of the process. This is due to the 
TABLE IV

COMPUTATIONAL REQUiREMENTS FOR THE MOM SOLUTION USING GMRES AND THE MLMDA SCHEME With TRIANGULAR BoXES $(f=9$ GHz). TAKING Advantage of Redundancies and the MultileVel Structure of the ObJect, THE Memory ReQuirements are Very SMaLl and Grow as $N$ While THE TIME PER ITERATION INCREASES ROUGHLY AS $N \log N$. OPTIMIZATION OF PRECONDITIONING IS THE OPEN POINT

\begin{tabular}{cccccccccc}
\hline & \multicolumn{2}{c}{ Building $[Z]$} & \multicolumn{2}{c}{ Preconditioning } & \multicolumn{2}{c}{ Iteration } & Total & Total \\
& Mem(MB) & $\mathrm{t}(\mathrm{s})$ & $\mathrm{Mem}(\mathrm{MB})$ & $\mathrm{t}(\mathrm{s})$ & n. it & txit(s) & It.time (s) & Mem.(MB) & Time (s) \\
\hline 4 it., $\mathrm{N}=568$ & 1.4 & 0.8 & 0.4 & 0.2 & 5 & 0.03 & 0.15 & 1.8 & 1.1 \\
5 it., $\mathrm{N}=1702$ & 4.1 & 2 & 3.8 & 1.5 & 4 & 0.17 & 0.68 & 7.9 & 4.1 \\
6 it., $\mathrm{N}=5104$ & 12.0 & 7 & 27.3 & 16 & 3 & 0.65 & 1.95 & 39.3 & 25 \\
7 it, $\mathrm{N}=15310$ & 38.2 & 19 & 128.3 & 133 & 9 & 2.60 & 23.4 & 166.5 & 176 \\
\hline
\end{tabular}

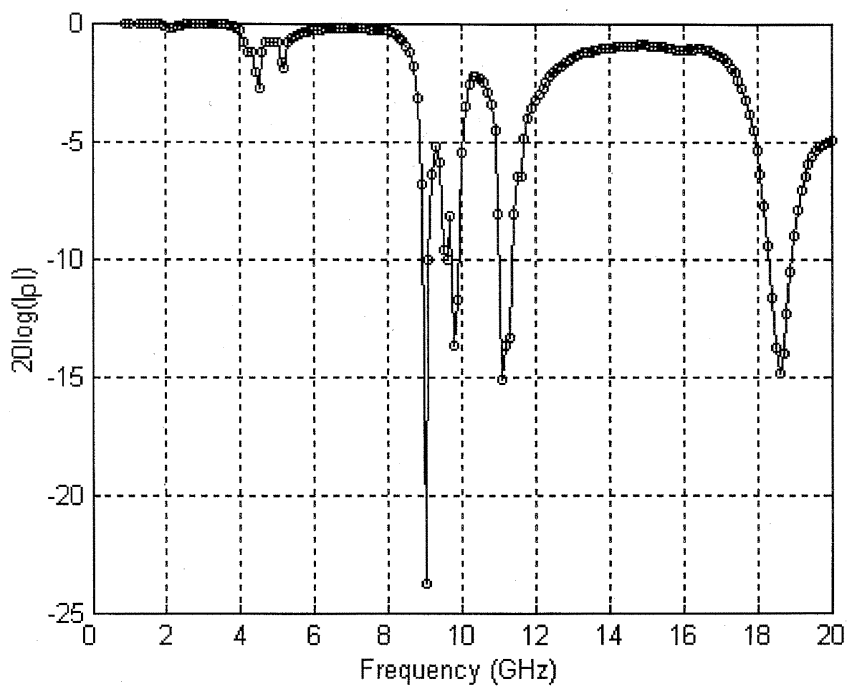

Fig. 4. Input reflection coefficient versus frequency for a seven iteration Sierpinski patch antenna.

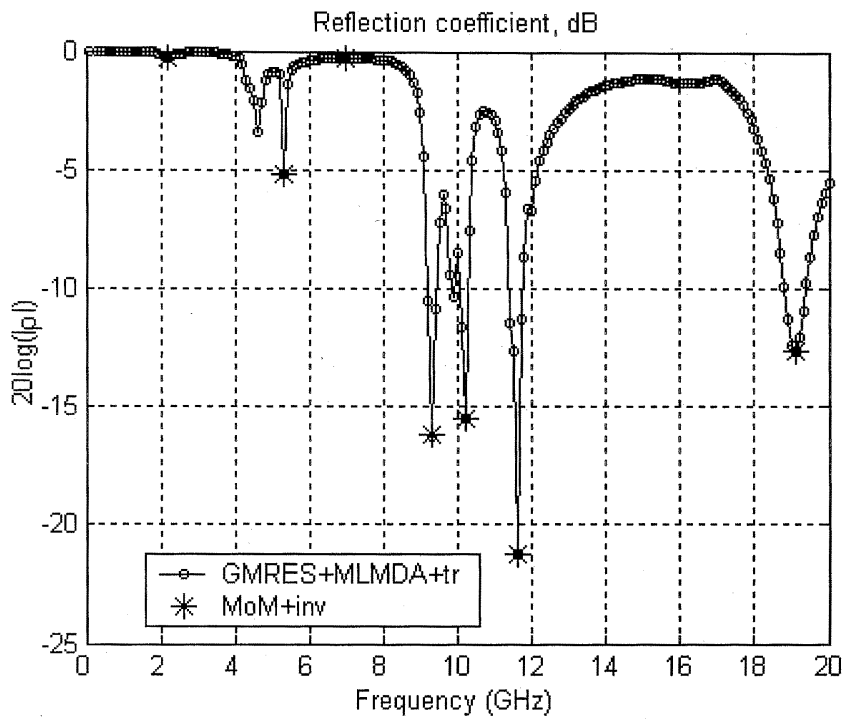

Fig. 5. Input reflection coefficient versus frequency for a six iteration Sierpinski patch antenna. It can be seen as there is not a noticeable loss of accuracy in the computation with GMRES + MLMDA with triangular boxes respect to the solution with direct inversion.

fact that it is only necessary to compute very few interactions between boxes.

The differences between the reference brute force approach of Table I and the optimized algorithm in Table IV are impressive, allowing us the analysis of this structure in a wide range of frequencies (Fig. 4). It must be pointed out that, for the problem of 15310 unknowns, the preconditioning, which has not been optimized for fractal structures yet, is taking most of the computational effort.

Finally, Fig. 5 shows the reflection coefficient of a six iteration Sierpinski antenna. The whole curve (200 frequencies) obtained with GMRES + MLMDA with triangular boxes is calculated in about the same time than seven specific frequencies with the standard MoM, without any noticeable loss of accuracy.

\section{CONCLUSION}

Computational requirements in the analysis of IFS defined pre-fractal antennas by MoM can be much reduced by making use of the redundancy and the multilevel structure of this geometry.

It has been shown that, for the case of a Sierpinski pre-fractal antenna, the combination of the GMRES and the MLMDA scheme, together with the appropriate choice of the shape of the boxes in the multilevel subdivision, leads to a very efficient solution. Our best implementation (Table IV) produces a reduction by a factor of 20 in the total computation time and a factor of 10 in the total memory, compared with a direct application of MoM (Table I). The bottleneck in the MoM analysis of IFS defined geometries is now in the preconditioning, which has not been optimized for IFS structures yet.

\section{ACKNOWLEDGMENT}

The authors wish to acknowledge also the support of EPFL for the visiting Prof. position of J. M. Rius at LEMA-EPFL from April 1 to June 30, 2001.

\section{REFERENCES}

[1] C. Puente, J. Romeu, R. Pous, X. García, and F. Benitez, "Fractal multiband antenna based on the sierpinski gasket," Electron. Lett., vol. 32, no. 1, pp. 1-2, Jan. 1996.

[2] C. Puente, J. Romeu, R. Pous, and A. Cardama, "On the behavior of the sierpinski multiband antenna," IEEE Trans. Antennas Propagat., vol. 46, pp. 517-524, Apr. 1998.

[3] R. F. Harington, Field Computation by Moment Methods. New York: MacMillan, 1968.

[4] Y. Saad, Iterative Methods for Sparse Linear Systems. Boston, MA: PWS, 1996.

[5] R. Coifman, V. Rohklin, and S. Wandzura, "The fast multipole method for the wave equation: A pedestrian description," IEEE Trans. Antennas Propagat., vol. 35, pp. 7-12, 1993.

[6] J. Song, C. C. Lu, and W. C. Chew, "Multilevel fast multipole algorithm for electromagnetic scattering by large complex objects," IEEE Trans. Antennas Propagat., vol. 45, pp. 1488-1493, Oct. 1997.

[7] E. Michelsen and A. Boag, "A multilevel matrix decomposition algorithm for analyzing scattering from large structures," IEEE Trans. Antennas Propagat., vol. 44, pp. 1086-1093, 1996. 
[8] J. Parrón, J. M. Rius, and J. R. Mosig, “Application of the multilevel matrix decomposition algorithm to the frequency analysis of large microstrip antenna arrays ," IEEE Trans. Magn., vol. 38, pp. 337-340, Mar. 2002.

[9] S. M. Rao, D. R. Wilton, and A. W. Glisson, "Electromagnetic scattering by surfaces of arbitrary shape," IEEE Trans. Antennas Propagat., vol. 30, pp. 409-419, May 1982.

[10] H. O. Pietgen, H. Jurgerns, and D. Saupe, Chaos and Fractals, New Frontiers in Science. New York: Springer Verlag, 1992.

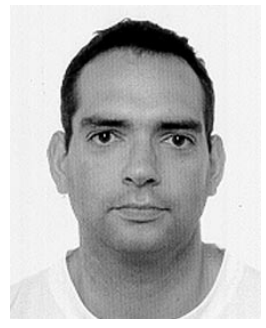

Josep Parrón was born in Sabadell (Barcelona), Spain, in 1970. He received the telecommunication engineer degree and the Doctor Ingeniero degree from the Politechnic University of Catalunya (UPC), Barcelona, Spain, in 1994 and 2001, respectively.

From September 2000 to May 2002 was an Assistant Professor at UPC. Since June 2002, he has been with the Universitat Autonoma de Barcelona (UAB) as an Associate Professor. He is the author of seven papers in international journals and 21 in international conference proceedings. His main research interests include fast algorithms for computational electromagnetics and their application to fractal antennas, multilayered mediums and superconductors.

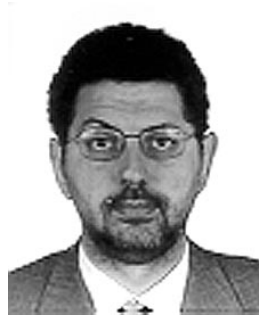

Jordi Romeu (S'88-M'93) was born in Barcelona, Spain, in 1962. He received the Ingeniero and Doctor Ingeniero degrees in Telecommunication Engineering, both from the Polytechnic University of Catalonia (UPC), Barcelona, Spain in 1986 and 1991, respectively.

In 1985, he joined the Electromagetic and Photonics Engineering Group of the Signal Theory and Communications Department, UPC where he is currently an Associate Professor engaged in research in antenna near field measurements, antenna diagnostics and antenna design. He was a Visiting Scholar at the Antenna Laboratory in the University of California, Los Angeles in 1999 with a NATO Scientific Program scholarship. He holds several patents and has published papers in the fields of antenna near-field measurements and diagnostics, and in antenna design.

Dr. Romeu was the Grand Winner of the 1998 European IT prize awarded by the European Commission for his contributions in the development of fractal antennas.

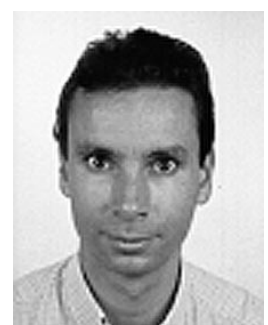

Juan M. Rius (S'89-M'91) received the "Ingeniero de Telecomunicación" degree in 1987 and the "Doctor Ingeniero" degree in 1991, both from the Universitat Politècnica de Catalunya (UPC), Barcelona, Spain.

In 1985 he joined the Electromagnetic and Photonic Engineering group at UPC, where currently holds a position of "Profesor Titular." He holds the position of "Visiting Professor" at the Swiss Federal Institute of Technology (EPFL), Lausanne, "CLUSTER Chair" at EPFL, and "Visiting Fellow" at City University of Hong Kong. From 1985 to 1988, he developed a new inverse scattering algorithm for microwave tomography in cylindrical geometry systems. He is the developer of the graphical electromagnetic computation (GRECO) approach for high-frequency RCS computation, the Integral equation formulation of the measured equation of invariance (IE-MEI) and the multilevel matrix decomposition algorithm in 3-D (MLMDA-3-D). $\mathrm{He}$ has 28 publications in refereed international journals and 54 in international conferences. Since 1989, he has been engaged in the research for new and efficient methods for numerical computation of electromagnetic scattering and (conventional or fractal) antennas radiation.

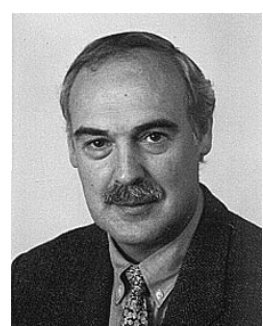

Juan R. Mosig (S'76-M'87-SM'94-F'99) was born in Cadiz, Spain. He received the electrical engineer degree from Universidad Politecnica de Madrid, Spain, in 1973 and the Ph.D. degree from the Laboratory of Electromagnetics and Acoustics at Ecole Polytechnique Fédérale de Lausanne (EPFL), Switzerland, in 1983.

In 1976, he joined the Laboratory of Electromagnetics and Acoustics at EPFL. Since 1991, he has been a Professor at EPFL and since 2000 the Head of the EPFL Laboratory of Electromagnetics and Acoustics (LEMA). In 1984, he was a Visiting Research Associate at Rochester Institute of Technology, Rochester, NY. He is the Chairman of a European COST project on antennas and is responsible for several research projects for the European Space Agency. He has also held scientific appointments at universities of Rennes (France), Nice (France), Technical University of Danemark and University of Colorado at Boulder. He is Coorganizer and Lecturer of yearly short intensive courses in numerical electromagnetics (Europe and USA). He is the author of four chapters in books on microstrip antennas and circuits and more than hundred reviewed papers. His research interests include electromagnetic theory, numerical methods and planar antennas.

Dr. Mosig is a Member of the Swiss Federal Commission for Space Applications. 